\title{
Biomarkers and balloons for the detection of Barrett oesophagus
}

\author{
A new study reports a biomarker-based \\ assay for detecting Barrett oesophagus \\ that does not rely on endoscopy. \\ The approach combines a swallowable \\ balloon device for oesophageal sampling \\ and sensitive and specific molecular \\ biomarkers (based on DNA methylation \\ states) for Barrett oesophagus. \\ Barrett oesophagus is a premalignant \\ metaplasia and recognized risk factor \\ for oesophageal adenocarcinoma. \\ The detection of Barrett oesophagus \\ usually relies on endoscopy (specifically \\ oesophagogastroduodenoscopy), \\ which is expensive, invasive and \\ not cost-effective for population \\ screening. In addition, aberrant cytosine \\ methylation within $\mathrm{CpG}$-rich genomic \\ islands accompanies many cancers, \\ and could act as a cancer biomarker. \\ Crucially, VIM DNA methylation \\ (mVIN) had previously been identified \\ as a sensitive Barrett oesophagus \\ biomarker. As such, Sanford Markowitz \\ and colleagues aimed to identify \\ methylated DNA biomarkers and to \\ improve the ease of oesophageal \\ sampling to develop disease detection \\ methods that could be used in screening \\ at-risk populations. \\ Using reduced representation bisulfite \\ sequencing analysis, the researchers \\ assessed oesophageal cancer biopsy \\ samples (alongside matched normal \\ squamous biopsy samples), biopsy or \\ brushing samples of Barrett oesophagus \\ and oesophageal cancer cell lines \\ to identify DNA methylation within \\ CpG-dense regions. High-frequency \\ methylation was identified within the \\ CCNA1 locus and this region was \\ the best candidate for discriminating \\ Barrett oesophagus lesions from \\ normal oesophagus.
}

Further testing of cytology brushings of the distal oesophagus ( $n=173$ ) confirmed CCNA1 DNA methylation (mCCNA1) as a Barrett oesophagus biomarker, distinguishing Barrett oesophagus-related metaplasia and neoplasia cases from normal individuals with an area under the curve of 0.95 . When mCCNA1 was combined with an established Barrett oesophagus biomarker (mVIM), the two-biomarker panel discriminated Barrett oesophagus with high accuracy ( $95 \%$ sensitivity and 91\% specificity). This high performance was repeated in an independent validation cohort of 149 individuals.

To move towards a non-endoscopic approach for the diagnosis of Barrett oesophagus, the researchers designed and engineered an encapsulated, inflatable, surface-textured balloon. The balloon was enclosed in a swallowable pill-sized capsule $(16 \times 9 \mathrm{~mm})$ attached to a thin catheter. Once delivered to the stomach, the balloon was inflated and gently withdrawn back to the distal oesophagus to sample the epithelial surface, after which the device was deflated, enclosed back into the capsule to protect the samples collected and then withdrawn and retrieved through the mouth. DNA was then extracted from the balloon surface for the $\mathrm{mVIM}$ and mCCNA1 biomarker analysis.

The device was tested in 156 individuals; 128 of these individuals were able to swallow the device. On average, the balloon reached the stomach within $3.3 \mathrm{~min}$ (range 1.0-7.7 min) with $72 \%$ of individuals reporting excellent tolerance. Importantly, adequate levels of DNA were obtained in $91 \%$ of the

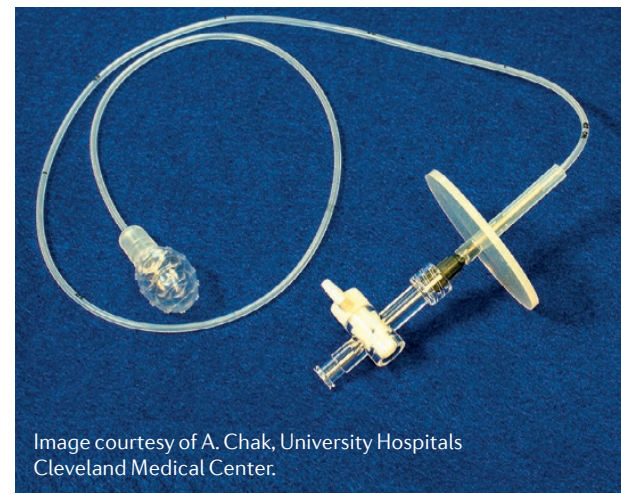

128 balloons processed and, in the 86 samples included in the analysis, mCCNA1 and mVIM discriminated cases from controls with similar high performance, sensitivity and specificity to cytology brushing samples.

"The combination of a DNA biomarker panel and a swallowable balloon for sampling the oesophagus creates a simple 5 min test with over $90 \%$ accuracy for identifying individuals who do or do not have Barrett oesophagus (that is, over $90 \%$ sensitivity and $90 \%$ specificity)," says Markowitz. "This first investigation ... studied a population that included many individuals already known to have Barrett oesophagus," explains co-senior author Amitabh Chak. "The next key test will be a clinical trial at multiple institutions and in a screening cohort of individuals who are not known to have or [known] not to have Barrett oesophagus."

Katrina Ray

ORIGINAL ARTICLE Moinova, H. R. et al. Identifying DNA methylation biomarkers for non-endoscopic detection of Barrett's esophagus. Sci. Transl. Med. 10, eaao5848 (2018) 